PROCEEDINGS OF THE

AMERICAN MATHEMATICAL SOCIETY

Volume 134, Number 5, Pages 1519-1525

S 0002-9939(05)08099-8

Article electronically published on October 6, 2005

\title{
BOREL SETS WITH COUNTABLE SECTIONS FOR NONSEPARABLE SPACES
}

\author{
PETR HOLICKÝ \\ (Communicated by Carl G. Jockusch, Jr.)
}

\begin{abstract}
We prove that every (extended) Borel subset $E$ of $X \times Y$, where $X$ is a complete metric and $Y$ is Polish, can be covered by countably many extended Borel graphs of mappings from $X$ to $Y$ if the sections $E_{x}=\{y \in Y$ : $(x, y) \in E\}, x \in X$, are countable. This is a nonseparable version of a classical theorem of Luzin and Novikov.
\end{abstract}

\section{INTRODUCTION}

We prove a nonseparable version of the theorem of Luzin and Novikov (see 9, Theorem 18.10] or [1, Theorem II.21]). The classical Luzin-Novikov theorem says that each Borel subset $B$ of the product of Polish spaces $X$ and $Y$ with countable sections $B_{x}=\{y \in Y:(x, y) \in B\}, x \in X$, can be covered by countably many Borel graphs of (Borel measurable) mappings from $X$ to $Y$ and that there is a Borel uniformization of $B$, i.e., a Borel graph in $B$ of a mapping defined on the whole projection of $B$ to $X$.

There is an abstract theorem of Burgess and Hillard (see, e.g., 1, Chapter IV, Section 4, Theorem 44]) generalizing the mentioned and some other significant theorems on coverings of sets with small sections.

Our result generalizes that of Luzin and Novikov to the case of nonseparable complete metric space $X$ and extended Borel sets $E \subset X \times Y$. Our proof does not use the Cantor derivatives of sections of $E$ as that in [1, Chapter II, Section 4] and thus it differs from the mentioned one even in the particular separable case. For yet another proof of the separable case see also [9, pp. 123-127].

\section{Souslin AND EXtended Borel Sets in METRIC SPACES}

The subset $S$ of a topological space $X$ is a Souslin set if

$$
S=\bigcup_{\left(n_{1}, n_{2}, \ldots\right) \in \mathbb{N}^{\mathbb{N}}} \bigcap_{k \in \mathbb{N}} F_{n_{1}, \ldots, n_{k}},
$$

where $F_{n_{1}, \ldots, n_{k}}$ are closed subsets of $X$.

Received by the editors September 27, 2004 and, in revised form, December 7, 2004.

2000 Mathematics Subject Classification. Primary 54H05; Secondary 54C65, 28A05.

Key words and phrases. Extended Borel sets, countable sections, nonseparable metric spaces.

This research was partially supported by grants GAČR 201/03/0933, GAČR 201/03/0931 and MSM 113200007.

(C)2005 American Mathematical Society 
If $S$ and its complement in $X$ are Souslin, we say that $S$ is a bi-Souslin set in $X$. Let us recall that Souslin subsets of Polish spaces are called analytic spaces and that bi-Souslin sets in an analytic space coincide with Borel subsets.

The class of extended Borel subsets of a metric space is the smallest class containing Borel sets that is a $\sigma$-field and that is closed under the unions of discrete families (see, e.g., 7]).

We are going to recall a few facts concerning Souslin and extended Borel sets in complete metric spaces. We need an important notion for the theory of nonseparable metric spaces introduced in $[5]$.

An indexed family $\left(M_{a} ; a \in A\right)$ of subsets of a metric space $X$ is discretely $\sigma$-decomposable ( $\sigma$-dd in short) if there are sets $M_{a n}, a \in A$ and $n \in \mathbb{N}$, such that $M_{a}=\bigcup_{n \in \mathbb{N}} M_{a n}$ and the indexed families

$$
\left(M_{a n}: a \in A\right), n \in \mathbb{N},
$$

are discrete in $X$, i.e., there is an open neighbourhood of $x$ which intersects $M_{a n}$ for at most one $a \in A$ whenever $x \in X$ and $n \in \mathbb{N}$. (It is not difficult to check that $\left(M_{a}: a \in A\right)$ is $\sigma$-dd iff it is point-countable as an indexed family and it has a $\sigma$-discrete network.) An (indexed) family $\mathcal{D}$ of subsets of $X$ is called relatively discrete if it is discrete in $\bigcup \mathcal{D}$.

We use without further reference the classical result of A. H. Stone that every metric space has a $\sigma$-discrete base (see, e.g., [2, Ch. 4, §4, Theorem 4]).

We need a lemma on projections along separable metric spaces. It essentially appeared in several previous papers. However, we do not know of a reference which would suit our present purpose exactly. Therefore, we also include its short proof.

Lemma 2.1. Let $X$ be a metric space, let $Y$ be a separable metric space, and let $\pi$ denote the projection mapping of $X \times Y$ onto $X$. If $\left(D_{a}: a \in A\right)$ is a relatively discrete indexed family of (extended Borel) subsets of $X \times Y$, then there are (extended Borel) sets $D_{a}(n) \subset X \times Y$ such that

$$
\begin{aligned}
& D_{a}=\bigcup_{n \in \mathbb{N}} D_{a}(n), \\
& D_{a}(n)=D_{a} \cap(P \times U) \text { for some open sets } P \text { in } X \text { and } U \text { in } Y \text {, and } \\
& \left(\pi\left(D_{a}(n)\right): a \in A\right) \text { is discrete for every } n \in \mathbb{N} \text {. }
\end{aligned}
$$

Proof. We fix a countable base $\left\{U_{m}: m \in \mathbb{N}\right\}$ of $Y$ and a base $\mathcal{C}=\bigcup_{k \in \mathbb{N}} \mathcal{C}_{k}$ of $X$ with all $\mathcal{C}_{k}$ discrete. There are open sets $G_{a} \supset D_{a}$ such that $G_{a} \cap D_{b}=\emptyset$ if $a \neq b$. We put $\mathcal{P}(a, k, m)=\left\{P \in \mathcal{C}_{k}: P \times U_{m} \subset G_{a},\left(P \times U_{m}\right) \cap D_{a} \neq \emptyset\right\}$. It is not difficult to observe that, for each fixed pair of $k$ and $m$ in $\mathbb{N}$, the families $\mathcal{P}(a, k, m)$, $a \in A$, are disjoint subfamilies of $\mathcal{C}_{k}$. We may also verify easily that the sets $D_{a}(k, m)=\left(\bigcup \mathcal{P}(a, k, m) \times U_{m}\right) \cap D_{a}, k, m \in \mathbb{N}$, form a countable decomposition of $D_{a}$. We get that $\pi\left(D_{a}(k, m)\right) \subset \bigcup \mathcal{P}(a, k, m)$ and, since $\mathcal{P}(a, k, m), a \in A$, are disjoint subfamilies of the discrete family $\mathcal{C}_{k}$ for every $k, m \in \mathbb{N}$, the indexed families $\left(\pi\left(D_{a}(k, m)\right): a \in A\right), k, m \in \mathbb{N}$, are also discrete. Finally, we may define $D_{a}(n)=D_{a}(k, m)$ if $n=\nu(k, m)$ for some bijection $\nu$ of $\mathbb{N} \times \mathbb{N}$ onto $\mathbb{N}$.

We formulate several equivalent descriptions of extended Borel subspaces of complete metric spaces in the following theorem. The characterizations (b), (c), and (d) were proved by Hansell in his fundamental papers [5, 6, 7] on descriptive theory of sets in nonseparable metric spaces. Condition (e) plays an important role in the proof of our main Theorem 3.1 and we did not find it in the literature. To prove that it is equivalent with (a), and with (b), we use the descriptions (c) and (d) 
below. We are going to use also a result from [3]. We could use another possibility to prove the necessity of (e) below and to show (a) implies (e) by showing (e) first for closed sets (which is trivial) and for open sets (which is a bit simpler than the general case). Then we might show and use that the smallest family containing closed and open sets which is closed under the operations of countable intersections, discrete unions, and countable disjoint unions is the family of all extended Borel sets in metric spaces. We prefer the first possibility as there is a paper with the needed result.

Theorem 2.2. Let $E$ be a subset of a complete metric space $X$. Then the following are equivalent:

(a) $E$ is extended Borel in $X$.

(b) $E$ is bi-Souslin in $X$.

(c) There is a closed set $F \subset D^{\mathbb{N}}$ for some discrete metric space $D$ and an injective continuous mapping $f: F \rightarrow E$ with $f(F)=E$ which maps discrete families of subsets of $F$ to $\sigma$-dd families in $E$.

(d) There is an extended Borel subset $F$ of a complete metric space and an injective continuous mapping $f: F \rightarrow E$ with $f(F)=E$ which maps discrete families of subsets of $F$ to $\sigma$-dd families in $E$.

(e) There is a closed set $G \subset X \times \mathbb{N}^{\mathbb{N}}$ such that the projection to $X$ restricted to $G$ is injective and it maps $G$ onto $E$.

Proof. The equivalence of (a) and (b) can be found in [6, Theorem 4.15 and Corollary 4.11], the equivalences of (a), (c), and (d) follow immediately from [7, Theorem 5.6 and Corollary 5.7]. The statement (e) implies (d) because every discrete family in $X \times \mathbb{N}^{\mathbb{N}}$ projects to a $\sigma$-dd family in $X$ by Lemma 2.1

To show that (c) implies (e), suppose that (c) holds. Note that (c) is a particular case of the condition $(\alpha)$ from [3, Theorem 4.1]. By [3, Theorem 4.1 $(\alpha$ implies $\delta)$ ] there is a Luzin sequence of covers of the (Luzin) space $E$. This means that there is a complete sequence $\left(\mathcal{C}_{n}\right)$ of $\sigma$-discrete covers of $E$ by subsets of $E$ such that $\left\{\bigcap_{k \in \mathbb{N}}{\overline{C_{1} \cap \cdots \cap C_{k}}}^{E}: C_{1} \in \mathcal{C}_{1}, \ldots, C_{k} \in \mathcal{C}_{k}\right\}$ is a partition of $E$.

Let us recall that $\left(\mathcal{C}_{n}\right)$ is complete in $E$ means that each filter $\mathcal{F}$ of subsets of $E$ which has nonempty intersection with $\mathcal{C}_{n}$ for every $n \in \mathbb{N}$ has an accumulation point, i.e., $\bigcap\left\{\bar{F}^{E}: F \in \mathcal{F}\right\} \neq \emptyset$.

We should remark that the discreteness considered in 3 was the uniform one, so even stronger than the topological one that we consider now. (Using the existence of a $\sigma$-discrete base of a metric space, we might achieve that the Luzin sequence $\left(\mathcal{C}_{n}\right)$ of covers of $E$ is such that the corresponding filters $\mathcal{F}$ as above have a unique accumulation point. However, we do not need this fact and so we are not going to explain it in detail.)

It is useful to realize that $\left(\mathcal{C}_{n}\right)$ is Luzin if and only if $\left(\mathcal{C}_{n}\right)$ is a complete sequence of $\sigma$-discrete covers and the $\mathcal{C}_{n}$ 's are partitions (disjoint covers) such that

$$
\bigcap_{n \in \mathbb{N}} C_{n}=\bigcap\left\{\overline{C_{1} \cap \cdots \cap C_{k}}: k \in \mathbb{N}\right\} \text { for } C_{n} \in \mathcal{C}_{n}
$$

as remarked in [3] after the definition of the notion of a Luzin sequence of covers.

To prove (e) we first use that each $\mathcal{C}_{k}$ is $\sigma$-discrete and find discrete families $\mathcal{C}_{k, n}$, $n \in \mathbb{N}$, such that $\mathcal{C}_{k}=\bigcup_{n \in \mathbb{N}} \mathcal{C}_{k, n}$ and the families $\mathcal{C}_{k, n}, n \in \mathbb{N}$, are pairwise disjoint. 
Given $n_{1}, \ldots, n_{k}$ from $\mathbb{N}$, we use the notation $\mathcal{U}_{n_{1}, \ldots, n_{k}}$ for the (discrete) family

$$
\left\{C_{1} \cap \cdots \cap C_{k}: C_{1} \in \mathcal{C}_{1, n_{1}}, \ldots, C_{k} \in \mathcal{C}_{k, n_{k}}\right\} .
$$

Finally, we put

$$
G=\bigcap_{k \in \mathbb{N}\left(n_{1}, \ldots, n_{k}\right) \in \mathbb{N}^{k}}\left(\overline{\bigcup_{\mathcal{U}_{1}, \ldots, n_{k}}} \times B\left(n_{1}, \ldots, n_{k}\right)\right)
$$

where

$$
B\left(n_{1}, \ldots, n_{k}\right)=\left\{\left(m_{i}\right) \in \mathbb{N}^{\mathbb{N}}:\left(m_{1}, \ldots, m_{k}\right)=\left(n_{1}, \ldots, n_{k}\right)\right\} .
$$

Clearly, the set $G$ is closed in $X \times \mathbb{N}^{\mathbb{N}}$. By (1) and discreteness of the $\mathcal{U}_{n_{1}, \ldots, n_{k}}$ 's, we have also that

$$
G=\bigcap_{k \in \mathbb{N}\left(n_{1}, \ldots, n_{k}\right) \in \mathbb{N}^{k}}\left(\bigcup \mathcal{U}_{n_{1}, \ldots, n_{k}} \times B\left(n_{1}, \ldots, n_{k}\right)\right) .
$$

We have that $\bigcup \mathcal{U}_{n_{1}, \ldots, n_{k}} \subset E$ and by (2) the projection of $G$ to $X$ is a subset of $E$. As $\mathcal{C}_{n}$ 's are covers, given $x \in E$ there is a (uniquely determined) sequence of elements $C_{1}$ of $\mathcal{C}_{1, n_{1}}, C_{2}$ of $\mathcal{C}_{2, n_{2}}$, etc. such that each of them contains $x$. Then $\left(x,\left(n_{1}, n_{2}, \ldots\right)\right) \in \bigcap_{k \in \mathbb{N}}\left(\left(C_{1} \cap \cdots \cap C_{k}\right) \times B\left(n_{1}, \ldots, n_{k}\right)\right) \subset G$. Hence, the set $E$ is the projection of $G$ to $X$.

Finally, if $\left(m_{1}, \ldots\right)$ and $\left(n_{1}, \ldots\right)$ are two distinct elements of $\mathbb{N}^{\mathbb{N}}$, then there is the smallest $i \in \mathbb{N}$ such that $m_{i} \neq n_{i}$. Let $\left(x,\left(n_{1}, \ldots\right)\right) \in G$. Then $x \notin \bigcup \mathcal{U}_{m_{1}, \ldots, m_{i}}$ and, using (2), we get that $G_{x}$ is a singleton. Thus we proved that (c) implies (e).

We point out a result on projections along separable spaces which follows easily from known facts.

Theorem 2.3. Let $E \subset X \times Y$ be an extended Borel set, with $X$ a complete metric and $Y$ Polish. If the sections $E_{x}, x \in X$ are countable, then the projection of $E$ to $X$ is extended Borel.

Proof. We may apply [4, Lemma 5.2] to the projection mapping $f$ of $X \times Y$ to $X$ restricted to $E$ because $f$ is continuous and takes discrete indexed families to $\sigma$-dd indexed families by Lemma 2.1. In this way we get that the projection $f(E)$ of $E$ is a complement of a Souslin set in $X$.

(To this end we might use also a separable reduction argument from [8]. This was done in $[8$, Theorem 5(c)] in a more general situation. We need to apply it here to the particular case, where the family of subsets of $Y$ that contain not more than one point stands for the hereditarily co-Souslin family $\mathcal{C}$.)

As the image of a Souslin subset $E$ of a complete metric space, i.e., of an absolutely analytic metric space under $f$, the projection of $E$ to $X$ is also Souslin in $X$ by [7, Corollary 4.2].

Finally, as a bi-Souslin set in a complete metric space, $f(E)$ is extended Borel by Theorem 2.2 .

\section{A nonseparable version of the theorem of Luzin and Novikov}

The main result of this section is the following improvement of the classical Luzin-Novikov theorem. 
Theorem 3.1. Let $E \subset X \times Y$ be an extended Borel subset of the product of a complete metric space $X$ and a Polish space $Y$ such that the sections $E_{x}=\{y \in$ $Y:(x, y) \in E\}, x \in X$, are at most countable.

Then there are countably many extended Borel graphs of mappings from $X$ to $Y$, which form a cover of $E$.

Remark 3.2. We should remark that, under the assumptions of Theorem 3.1, there is even a countable cover of $E$ by extended Borel uniformizations $U_{n}, n \in \mathbb{N}$, of $E$. Indeed, let $G_{n}$ be the extended Borel graphs which exist due to Theorem 3.1. We denote the projection mapping of $X \times Y$ to $X$ by $\pi$ and define $U_{n}=G_{n} \cup \bigcup\left\{G_{k} \backslash\right.$ $\bigcup\left\{\pi\left(G_{i}\right) \times Y: i<k\right.$ or $\left.\left.i=n\right\}: k \neq n\right\}$. Then the sets $U_{n}$ form a sequence of uniformizations which covers E. Each $U_{n}$ is extended Borel due to Theorem 2.3. Thus our theorem, together with Theorem 2.3, gives also an improvement of the original Novikov theorem on the Borel uniformization (see [10, §9]).

We show first that we may suppose, without loss of generality, that $E$ is closed.

Lemma 3.3. If the claim of Theorem 3.1 holds for closed sets $E \subset X \times Y$ with countable sections $E_{x}, x \in X$, for all complete metric spaces $X$ and Polish spaces $Y$, then Theorem 3.1 holds.

Proof. Let $E$ be an arbitrary extended Borel subset of $X \times Y$ which has countable sections $E_{x}, x \in X$. By the above Theorem 2.2, there is a closed set $F \subset(X \times$ $Y) \times \mathbb{N}^{\mathbb{N}}$ such that the projection of $F$ to $X \times Y$ is injective and maps $F$ onto $E$. The space $Z=Y \times \mathbb{N}^{\mathbb{N}}$ is Polish and the sections $F_{x} \subset Z, x \in X$, of $F \subset X \times Z$ are countable. The conclusion of Theorem 3.1 for $F \subset X \times Z$ holds by our assumption and so there are countably many extended Borel graphs $H_{k}, k \in \mathbb{N}$, of mappings from $X$ to $Z$ which cover $F$. The projections $G_{k}, k \in \mathbb{N}$, to $X \times Y$ of $H_{k}, k \in \mathbb{N}$, are extended Borel by Theorem 2.3 since the sections $\left(H_{k}\right)_{(x, y)},(x, y) \in X \times Y$, are countable (they even contain at most one point). Each $G_{k}$ is the graph of a mapping from $X$ to $Y$ as $H_{k}$ was a graph of a mapping from $X$ to $Y \times \mathbb{N}^{\mathbb{N}}$, and as they form a cover of $E$, this concludes the proof of our lemma.

According to Lemma 2.1 we may easily realize that it is sufficient to look for a $\sigma$-discrete family of extended Borel graphs instead of a countable family when proving Theorem 3.1 .

From now on suppose that $X$ and $Y$ are as in Theorem 3.1. Put $\mathcal{C}_{0}=\{Y\}$ and $\mathcal{D}_{0}=\{X\}$. Let $\mathcal{C}_{n}$ denote a fixed countable base of $Y$ consisting of open sets of diameter at most $\frac{1}{2^{n}}$ for $n=1, \ldots$ Similarly, let $\mathcal{D}_{n}$ denote a $\sigma$-discrete base of $X$ consisting of open sets of diameter at most $\frac{1}{2^{n}}$ for $n=1, \ldots$

We say that a family $\mathcal{U}=\left\{U_{i_{1}, \ldots, i_{n}}:\left(i_{1}, \ldots, i_{n}\right) \in\{0,1\}^{n}\right\} \subset \mathcal{C}_{n}$ is $n$-admissible for $n=0,1, \ldots$ if its elements have pairwise disjoint closures.

Now we prove, in a more or less standard way, a lemma which recalls the basic step when using the procedure of applying Cantor derivatives to the sections of $E$ (cf. [1]).

Lemma 3.4. Let $E$ be an extended Borel subset of $X \times Y$ with countable sections $E_{x}, x \in X$. Then the set $G=\left\{(x, y) \in E:\{y\}=E_{x}\right\}$ is extended Borel in $X \times Y$.

Proof. Let $\mathcal{C}$ be a countable base for $Y$. Then the image of $G$ under the projection mapping $\pi$ of $X \times Y$ to $X$ is equal to

$$
\pi(E) \backslash \bigcup\left\{\bigcap_{i=0,1} \pi\left(E \cap\left(X \times U_{i}\right)\right): U_{i} \in \mathcal{C}, i=0,1, U_{0} \cap U_{1}=\emptyset\right\} .
$$


All the projections involved are extended Borel by Theorem 2.3. Thus $G=(\pi(G) \times$ $Y) \cap E$ is extended Borel.

We introduce now the key notion for our proof of Theorem 3.1 Further on, we suppose that $E \subset X \times Y$ is as in Theorem 3.1

Definition 3.5. Let $P \in \mathcal{D}_{n}$ and $\mathcal{U}=\left\{U_{i_{1}, \ldots, i_{n}}:\left(i_{1}, \ldots, i_{n}\right) \in\{0,1\}^{n}\right\} \subset \mathcal{C}_{n}$ be $n$-admissible. We say that $E$ is $\mathcal{U}$-coverable over $P$ if there is a $\sigma$-discrete family of extended Borel graphs $G_{a}, a \in A$, of mappings from $X$ to $Y$ such that for every $x \in P$ there is an $\left(i_{1}, \ldots, i_{n}\right) \in\{0,1\}^{n}$ with

$$
\left(E \backslash \bigcup_{a \in A} G_{a}\right)_{x} \cap U_{i_{1}, \ldots, i_{n}}=\emptyset .
$$

We say that the family $\left\{G_{a}: a \in A\right\}$ demonstrates that $E$ is $\mathcal{U}$-coverable over $P$ in such a case.

The following lemma is the main observation making the later inductive procedure possible.

Lemma 3.6. Let $\mathcal{U} \subset \mathcal{C}_{n}$ be $n$-admissible and $P \in \mathcal{D}_{n}$. Further, let $E$ be $\mathcal{W}$ coverable over $Q$ for every $Q \in \mathcal{D}_{n+1}$ and for every $(n+1)$-admissible family $\mathcal{W}=\left\{W_{i_{1}, \ldots, i_{n+1}}:\left(i_{1}, \ldots, i_{n+1}\right) \in\{0,1\}^{n+1}\right\} \subset \mathcal{C}_{n+1}$ which satisfy that $\bar{Q} \subset P$ and $\overline{W_{i_{1}, \ldots, i_{n+1}}} \subset U_{i_{1}, \ldots, i_{n}}$. (We say that $(Q, \mathcal{W})$ is compatible with $(P, \mathcal{U})$ if the above relations hold.)

Then $E$ is $\mathcal{U}$-coverable over $P$.

Proof. Let $E(Q, \mathcal{W})$ be the union of a $\sigma$-discrete family of extended Borel graphs which demonstrates that $E$ is $\mathcal{W}$-coverable over $Q$ for every $(Q, \mathcal{W})$ compatible with $(P, \mathcal{U})$. We may and do suppose that the extended Borel graphs from $E(Q, \mathcal{W})$ are subsets of $Q \times Y$. The set $\mathcal{A}(Q)=\{\mathcal{W}:(Q, \mathcal{W})$ compatible with $(P, \mathcal{U})\}$ is a set of finite subfamilies of the countable family $\mathcal{C}_{n+1}$, and so it is countable. Thus $\bigcup\{E(Q, \mathcal{W}): \mathcal{W} \in \mathcal{A}(Q)\}$ can be covered by a $\sigma$-discrete family of extended Borel graphs. Since the family $\mathcal{D}_{n+1}$ is $\sigma$-discrete, and each graph from $E(Q, \mathcal{W})$ is a subset of $Q \times Y$, we may easily verify that the set

$$
E_{0}=\bigcup_{Q \in \mathcal{D}_{n+1}} \bigcup_{\mathcal{W} \in \mathcal{A}(Q)} E(Q, \mathcal{W})
$$

can be covered by a $\sigma$-discrete family of extended Borel graphs.

Consider the section $\left(E \backslash E_{0}\right)_{x}$ for any $x \in P$. We find a set $Q$ such that $x \in Q \in$ $\mathcal{D}_{n+1}, \bar{Q} \subset P$. Let $\mathcal{U}=\left\{U_{i_{1}, \ldots, i_{n}}:\left(i_{1}, \ldots, i_{n}\right) \in\{0,1\}^{n}\right\}$. If each $\left(E \backslash E_{0}\right)_{x} \cap U_{i_{1}, \ldots, i_{n}}$ contains two distinct points, say $y_{i_{1}, \ldots, i_{n}, 0}$ and $y_{i_{1}, \ldots, i_{n}, 1}$, we find $\mathcal{W}=\left\{U_{i_{1}, \ldots, i_{n+1}}\right.$ : $\left.\left(i_{1}, \ldots, i_{n+1}\right) \in\{0,1\}^{n+1}\right\}$ which is $(n+1)$-admissible so that $(Q, \mathcal{W})$ is compatible with $(P, \mathcal{U})$ and $y_{i_{1}, \ldots, i_{n+1}} \in U_{i_{1}, \ldots, i_{n+1}}$. Then $(E \backslash E(Q, \mathcal{W}))_{x} \cap U_{i_{1}, \ldots, i_{n+1}}=\emptyset$ for some $\left(i_{1}, \ldots, i_{n+1}\right)$. Hence $E(Q, \mathcal{W})$ contains $\left(x, y_{i_{1}, \ldots, i_{n+1}}\right)$, which contradicts the choice of $y_{i_{1}, \ldots, i_{n+1}}$ and the definition of $E_{0}$. Thus

$$
(\forall x \in P)\left(\exists\left(i_{1}, \ldots, i_{n}\right) \in\{0,1\}^{n}\right)\left(\operatorname{card}\left(\left(E \backslash E_{0}\right)_{x} \cap U_{i_{1}, \ldots, i_{n}}\right) \leq 1\right),
$$

where card $A$ stands for the cardinality of the set $A$.

Consider the set $\left(E \backslash E_{0}\right) \cap\left(P \times U_{i_{1}, \ldots, i_{n}}\right)$ for each $\left(i_{1}, \ldots, i_{n}\right) \in\{0,1\}^{n}$. By Lemma 3.4 each of the finitely many sets $\left\{(x, y): x \in P,\{y\}=\left(\left(E \backslash E_{0}\right) \cap\right.\right.$ $\left.\left.\left(P \times U_{i_{1}, \ldots, i_{n}}\right)\right)_{x}\right\}$ is an extended Borel graph. Finally, these graphs together with 
$E_{0}$ can be covered by a $\sigma$-discrete family of extended Borel graphs which, by (3), demonstrates that $E$ is $\mathcal{U}$-coverable over $P$.

Proof of Theorem 3.1. Due to Lemma 3.3 we may and do assume that the set $E$ is closed. Suppose that the conclusion of the theorem does not hold. According to Lemma 2.1. we know that $E$ cannot be covered by a $\sigma$-discrete family of extended Borel graphs. In other words, $E$ is not $\mathcal{C}_{0}$-coverable over $X$. Put $P_{0}=X \in \mathcal{D}_{0}$ and $U_{\emptyset}=Y \in \mathcal{C}_{0}$. Using Lemma 3.6 inductively, we get also for each $n \geq 1$ a set $P_{n} \in \mathcal{D}_{n}$ and a family of sets $\left\{U_{i_{1}, \ldots, i_{n}}:\left(i_{1}, \ldots, i_{n}\right) \in\{0,1\}^{n}\right\} \subset \mathcal{C}_{n}$ such that

$$
\begin{aligned}
& \overline{P_{n}} \subset P_{n-1}, \overline{U_{i_{1}, \ldots, i_{n}}} \subset U_{i_{1}, \ldots, i_{n-1}}, \\
& \overline{U_{i_{1}, \ldots, i_{n-1}, 0}} \cap \overline{U_{i_{1}, \ldots, i_{n-1}, 1}}=\emptyset \text {, and }
\end{aligned}
$$

$E$ is not $\mathcal{U}_{n}=\left\{U_{i_{1}, \ldots, i_{n}}:\left(i_{1}, \ldots, i_{n}\right) \in\{0,1\}^{n}\right\}$-coverable over $P_{n}$.

Then the completeness of $X$ and the choice of the $P_{n}$ 's ensure that there is an $x \in X$ such that $\{x\}=\bigcap_{n \in \mathbb{N}} P_{n}$. Moreover, using the completeness of both, $X$ and $Y$, and the choice of the $U_{i_{1}, \ldots, i_{n}}$ 's as well, we come to the existence of $y_{\iota} \in Y$ for every $\iota=\left(i_{1}, i_{2}, \ldots\right) \in\{0,1\}^{\mathbb{N}}$ such that $\left\{\left(x, y_{\iota}\right)\right\}=\bigcap_{n \in \mathbb{N}}\left(\overline{P_{n}} \times \overline{U_{i_{1}, \ldots, i_{n}}}\right) \cap E$. Here we use that all the sets $\left(P_{n} \times U_{i_{1}, \ldots, i_{n}}\right) \cap E$ are nonempty because otherwise $E$ is $\mathcal{U}_{n}$-coverable over $P_{n}$ (by an empty family of graphs) and that $E$ is closed. It follows also that the set $\left\{\left(x, y_{\iota}\right): \iota \in\{0,1\}^{\mathbb{N}}\right\}$ is a homeomorphic copy of the Cantor, in particular uncountable, set contained in $E_{x}$. This is a contradiction with our assumption on the cardinality of sections $E_{x}$.

\section{ACKNOWLEDGMENTS}

The author thanks M. Zelený and J. Pelant for their useful remarks.

\section{REFERENCES}

[1] C. Dellacherie, Un cours sur les ensembles analytiques, in: Analytic sets, C.A.Rogers, Academic Press, London 1980, pp. 183-316.

[2] R. Engelking, Outline of General Topology, North-Holland, Amsterdam, 1968. MR0230273 (37:5836)

[3] Z. Frolík and P. Holický, Analytic and Luzin spaces (non-separable case), Topology Appl. 19 (1985), 129-156. MR0789594 (86m:54052)

[4] Z. Frolík and P. Holický, Applications of Luzinian separation principles (non-separable case), Fund. Math. 117 (1983), 165-185. MR0719837 (85k:54044)

[5] R. W. Hansell, Borel measurable mappings for nonseparable metric spaces, Trans. Amer. Math. Soc. 161 (1971), 145-169. MR0288228 (44:5426)

[6] R. W. Hansell, On the non-separable theory of $k$-Borel and $k$-Souslin sets, General Topology and Appl. 3 (1973), 161-195. MR0319170 (47:7716)

[7] R. W. Hansell, On characterizing non-separable analytic and extended Borel sets as types of continuous images, Proc. London Math. Soc. 28 (1974), 683-699. MR0362269 (50:14711)

[8] P. Holický and V. Komínek, On projections of nonseparable Souslin and Borel sets along separable spaces, Acta Univ. Carolin. Math. Phys. 42 (2001), 33-41. MR1900390 (2003c:54070)

[9] A. S. Kechris, Classical Descriptive Set Theory, Springer-Verlag, New York, 1995. MR.1321597 (96e:03057)

[10] P. Novikoff, Sur les fonctions implicites mesurables B, Fund. Math. 17 (1931), 8-25.

Department of Mathematical Analysis, Charles University, Sokolovská 83, 18675 Prague 8, Czech Republic

E-mail address: holicky@karlin.mff.cuni.cz 